\section{PARALLEL MONTE CARLO SIMULATION USING DESKTOP COMPUTERS}

John Henry J. Scott*, Robert L. Myklebust**, Dale E. Newbury* *National Institute of Standards and Technology **Myklebust Consulting

Monte Carlo simulation of electron scattering in solids has proven valuable to electron microscopists for many years. ${ }^{1}$ The electron trajectories, $x$-ray generation volumes, and scattered electron signals produced by these simulations are used in quantitative $x$-ray microanalysis, image interpretation, experimental design, and hypothesis testing. Unfortunately, these simulations are often computationally expensive, especially when used to simulate an image or survey a multidimensional region of parameter space.

Here we present techniques for performing Monte Carlo simulations in parallel on a cluster of existing desktop computers. The simulation of multiple, independent electron trajectories in a sample and the collateral calculation of detected $x$ ray and electron signals fall into a class of computational problems termed "embarrassingly parallel", since no information needs to be exchanged between parallel threads of execution during the calculation. Such problems are ideally suited to parallel multicomputers, where a manager process distributes the computational burden over a large number of nodes. The utility of this scheme in the context of electron microscopy has been elegantly demonstrated using dedicated, homogeneous supercomputers. ${ }^{2-4}$ Significant speedups are seen when existing Monte Carlo codes are adapted for use on vector supercomputers such as the CRAY-YMP ${ }^{\#}$ and even more dramatic gains are seen when massively parallel supercomputers such as the nCUBE and Intel Paragon are used. ${ }^{3}$ In this work, we achieve similar speedups using a heterogeneous collection of desktop personal computers linked by conventional TCPIIP networks. The Monte Carlo simulation runs transparently in the background and the machines remain free for other tasks such as word processing, data analysis, or instrument control. This solution should be particularly appealing to academic users, small businesses, and similar groups who enjoy access to a growing pool of PCs, but lack easy access to a supercomputer.

Adaptation of existing Monte Carlo programs for parallel execution can be achieved using any of several off-the-shelf message passing toolkits. As a demonstration, we used the Parallel Virtual Machine ${ }^{5}$ library to enhance the NBS Monte Carlo code ${ }^{6}$ to run on a distributed multicomputer. In our group, virtual machines have been assembled that simultaneously contain hosts running Windows 95, Windows NT, Linux, and SunOS. Toolkits such as LAM or MPICH that conform to the Message Passing Interface (MPI) standard can also be used. The modifications consist of partitioning the code into two units, a manager and a worker, and embedding function calls from the library to marshal the virtual machine and handle the exchange of data. The manager process collects input from the user, spawns multiple instances of the worker process on the various nodes of the virtual machine, multicasts the initial problem description to the collective, and merges the results sent back from the workers. The many (typically identical) worker processes do most of the work.

To get reproducible timing data and to observe the scaling properties of the implementation, our example code was run on an unloaded cluster of 16 Pentium II nodes running Linux. Fig. 1

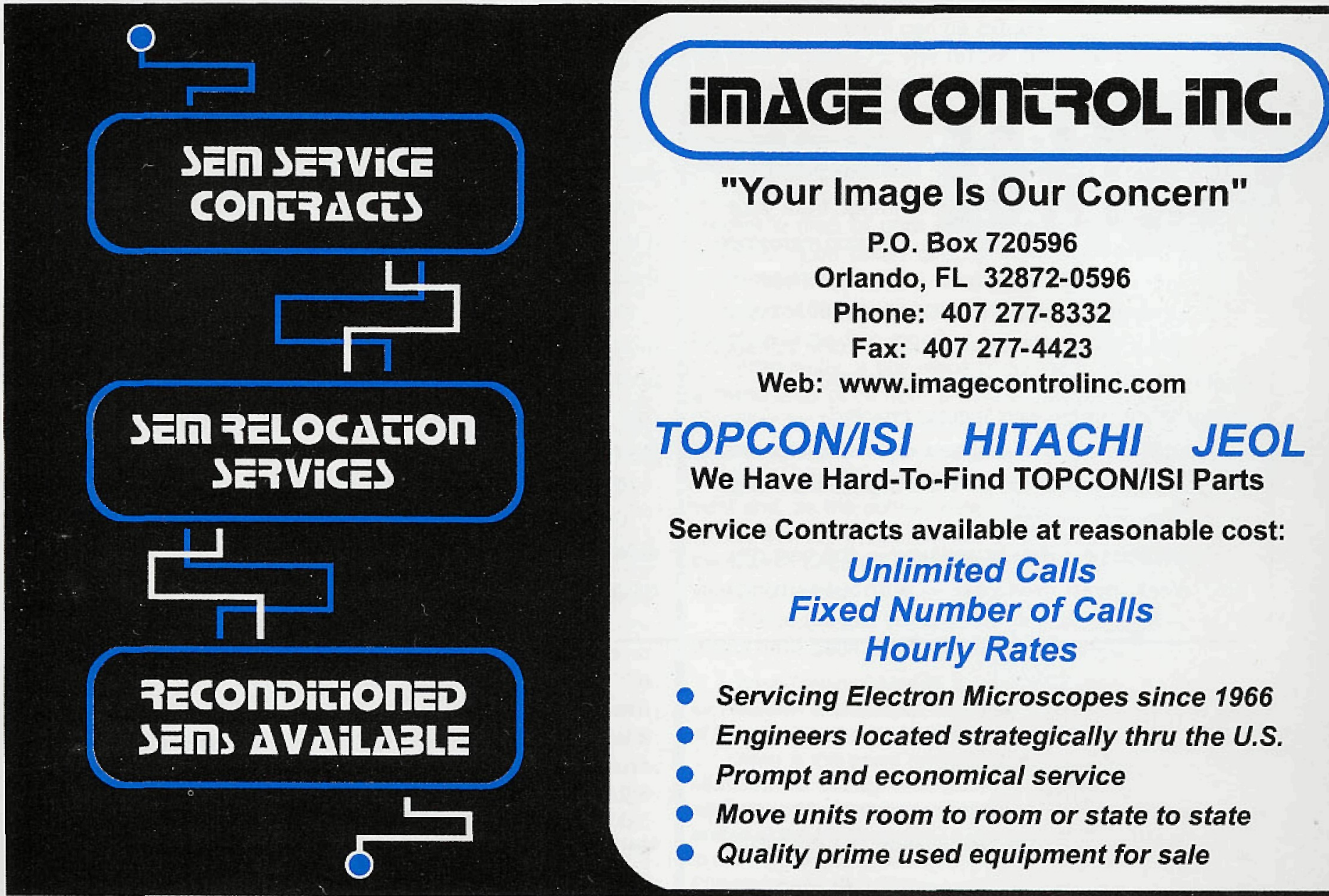


shows the wall clock time needed to complete each run and the estimated throughput of the virtual machine in electrons/second. With 16 nodes, the virtual machine processed 160,000 electrons in 64 seconds, or about 2,500 electrons $/ \mathrm{s}$. This compares favorably with speeds achieved by dedicated supercomputers. ${ }^{3}$

References

I. D.C. Joy, Monte Carlo Modeling for Electron Microscopy and Microanalysis, Oxford (1995).

2. A. D. Romig, Jr. et al., in J. R. Michael et al, Eds., Microbeam Analysis - 1990, San Francisco Press (1990) 275.

3. J. R. Michael et al., Uitramicroscopy 51 (1993) 160.

4. A. D. Romig et al., in G. W. Bailey et al., Eds., Proc. $52^{\text {nd }}$ Ann. Mtg. MSA/29 th Ann. Mtg. MAS (1994)910.

5. PVM is free, and can be obtained from http://www.epm.ornl. gov/pvm/.

6. R. L. Myklebust et al., in Use of Monte Carlo Calculations in Electron Probe Microanalysis and Scanning Electron Microscopy, Washington, D.C., NBS Special Pub 460 (1975)105.

JHS gratefully acknowledges the help and advice of Judy Devaney, Michael Indovina, and Carl Spangler of the NIST High Performance Systems and Services Division.

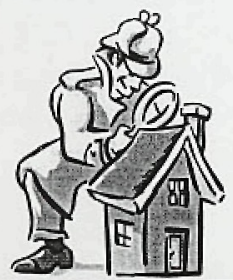

Calculation time and speed

bulk Fe, $20 \mathrm{keV}, 160,000$ electrons each node: $333 \mathrm{MHz}$ Pentium II

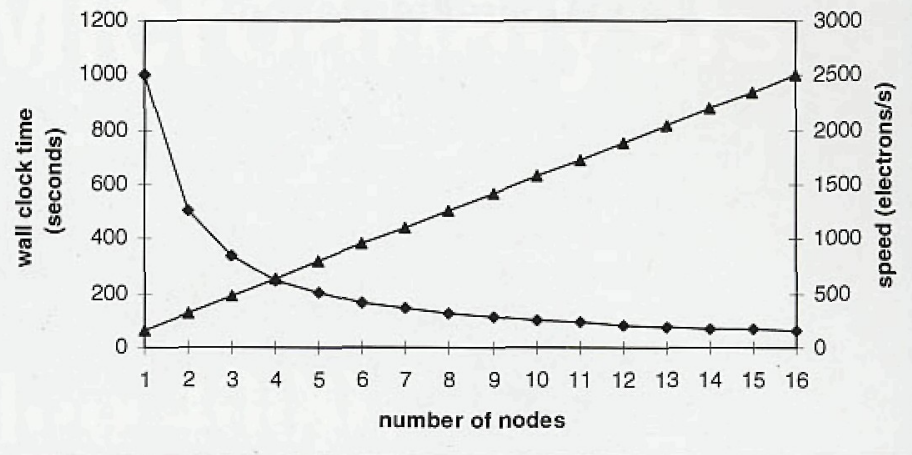

FIG. 1. Plot of the elapsed time of calculation measured in seconds (left axis), and speed of calculation in electrons/s (right axis) as a function of the number of nodes used for the simulation. Each node consisted of a $333 \mathrm{MHz}$ Pentium II processor with 256 MB of RAM. Message passing was implemented using Parallel Virtual Machine v3.3. 11 over a $100 \mathrm{Mbit} / \mathrm{s}$ Ethernet network.

Certain commercial equipment, instruments, or materials are identified in this report to specify adequately the experimental procedure. Such identification does not imply recommendation or endorsement by the National Institute of Standards and Technology, nor does it imply that the materials or equipment identified are necessarily the best available for the purpose.

\section{Si(Li)-Detectors}

stainless steel dewar, UHV technology excellent energy resolution best choice for light element analysis unrivalled long term stability

\section{Microanalysis Systems}

slandardless andysis on rough surfaces and particles physical calculation of the bremssiratilung integrated sexpert system $\alpha$ comprehensive error calculation application-specific automatic quantification
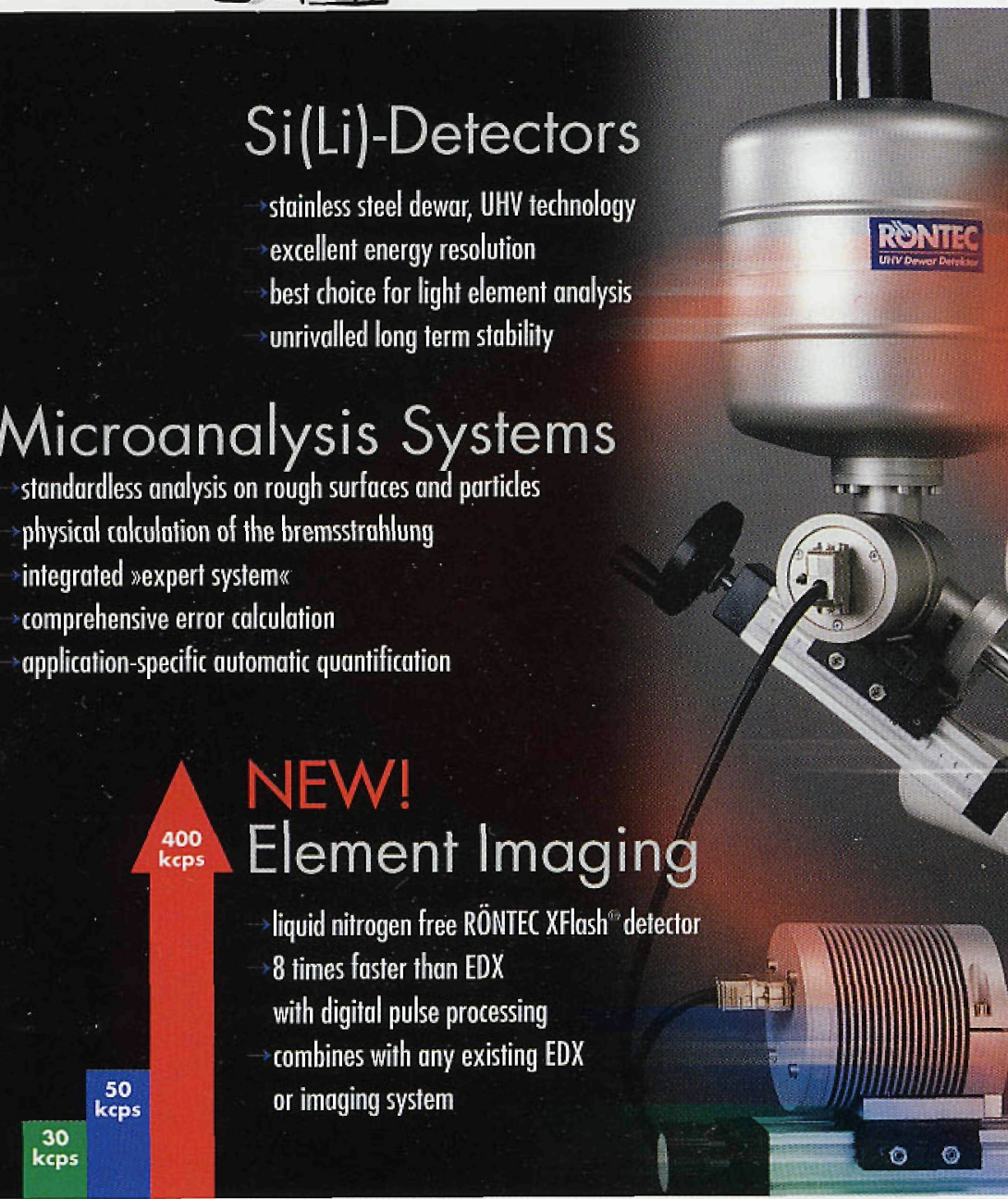

A

liquid nitrogen free RÖNTEC XFlash" detecior 8 times faster than EDX with digital pulse processing combines with any existing EDX or imaging system
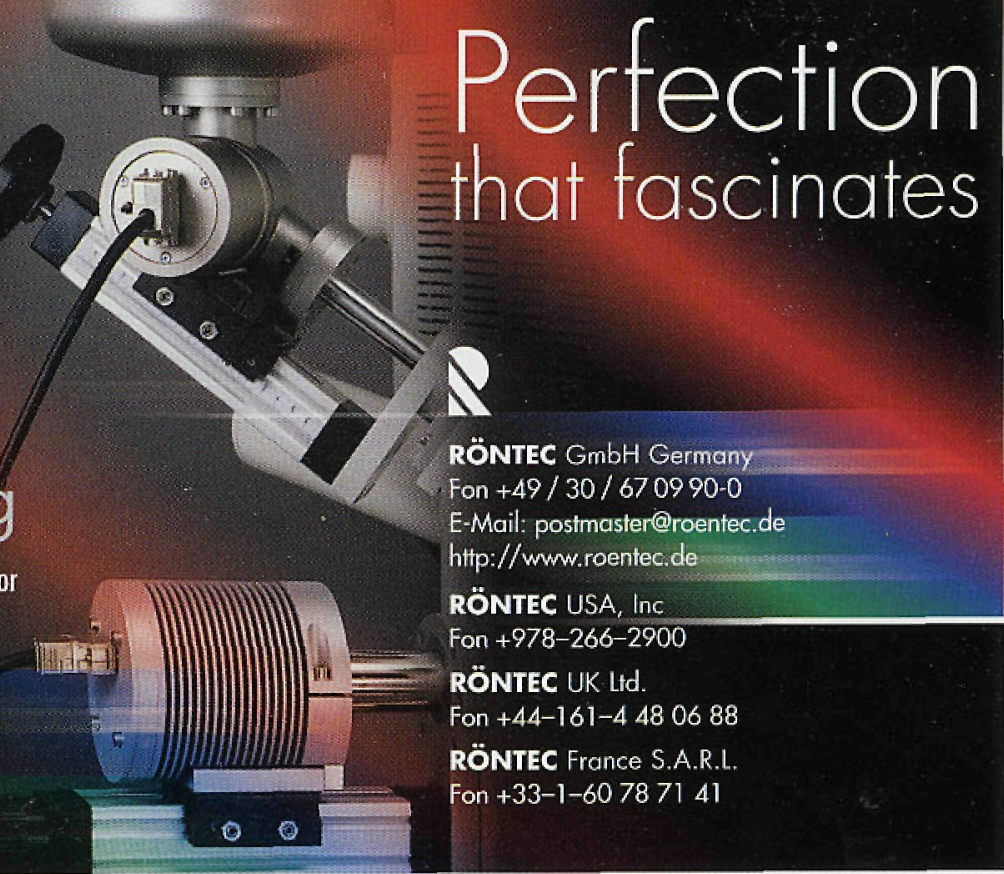